\title{
L-band cryogenic radio astronomy receiver front-end of the Square Kilometre Array
}

\author{
Robert Lehmensiek $\odot,{ }^{\text {a,b, } * \text { Ockert B. Jacobs, }{ }^{\mathrm{a}} \text { Nianhua Jiang, }}{ }^{\mathrm{c}}$ \\ Evan C. Knox-Davies, ${ }^{a}$ Sarel J. Marais, ${ }^{a}$ and Isak P. Theron $\odot^{a}$ \\ ${ }^{a}$ EMSS Antennas (Pty) Ltd, Stellenbosch, South Africa \\ ${ }^{b}$ University of Stellenbosch, Stellenbosch, South Africa \\ ${ }^{c}$ Herzberg Astronomy and Astrophysics Research Center, Victoria, British Columbia, Canada
}

\begin{abstract}
This paper describes the design and measured performance of the band 2 (L-band, $950 \mathrm{MHz}-1760 \mathrm{MHz}$ ) cryogenic receiver front-end of the Square Kilometre Array (SKA) radio telescope dish array. The system comprises a wide flare-angle axially corrugated conical horn, a dual linearly polarized orthogonal mode transduce, a noise injection directional coupler, and two amplification stages. Its compact design and cryogenic cooling allow for a very low receiver noise temperature, and it presents another step in the continuous improvement of the noise temperature performance. A Gifford-McMahon cooler physically cools the OMT with its integrated directional coupler to around $70 \mathrm{~K}$, and the first stage low-noise amplifier to about $15 \mathrm{~K}$. A bespoke measurement setup was designed to measure the system's performance. The measured receiver noise is about $6 \mathrm{~K}$ across the frequency band. () The Authors. Published by SPIE under a Creative Commons Attribution 4.0 International License. Distribution or reproduction of this work in whole or in part requires full attribution of the original publication, including its DOI. [DOI: 10.1117/1.JATIS.8.1.011002]
\end{abstract}

Keywords: antenna feeds; cryogenics; horn antennas; orthogonal mode transducer; low-noise amplifier; receivers; wideband.

Paper 21058SS received May 19, 2021; accepted for publication Jun. 25, 2021; published online Oct. 22, 2021.

\section{Introduction}

Receiving sensitivity, arguably the most important metric for astronomical radio telescopes, is defined as the ratio of effective aperture area to system noise temperature $\left(A_{\mathrm{e}} / T_{\text {sys }}\right)$. The system noise temperature is the sum of the antenna noise temperature, determined by the feed horn and reflector system's combined radiation characteristics, and the receiver noise temperature, determined by resistive losses in the receiver chain plus the low-noise amplifier's (LNA) noise contribution. The antenna noise temperature includes the emission from water vapor and dry air in the atmosphere, the apparent temperature due to the cosmic microwave background and galactic emissions as seen through the atmosphere, and the ground emission. Since the sensitivity scales linearly with the total collecting area of the system, any improvement to the noise temperature of a single dish in an interferometric array leads to a corresponding reduction in the total number of dishes required to achieve the same system sensitivity. With a $15 \mathrm{~K}$ system temperature, a $0.5 \mathrm{~K}$ improvement in the system temperature allows removing one dish in thirty, saving about $7 \%$ in operational time for each science experiment, highlighting the importance of a carefully optimized antenna system and cryogenic receiver front-end.

The mid-frequency array of the Square Kilometer Array (SKA) radio telescope will consist of $15-\mathrm{m}$ projected diameter shaped offset Gregorian reflector antennas. ${ }^{1}$ The current frequency band, $350 \mathrm{MHz}$ to $15.4 \mathrm{GHz}$, will be covered by an indexer mechanism mounted with six receivers: five $60 \%$ bandwidth receivers, and one $3: 1$ band receiver. All of the receivers cause a single boresight beam per antenna.

Each receiver collects electromagnetic (EM) radiation concentrated at the focus of the reflector system and transforms it into electrical signals for both of the received (linear) orthogonal polarizations. The L-band receiver covers the frequency range from 950 to $1760 \mathrm{MHz}(\sim 60 \%$

*Address all correspondence to Robert Lehmensiek, lehmensk@emss.co.za 


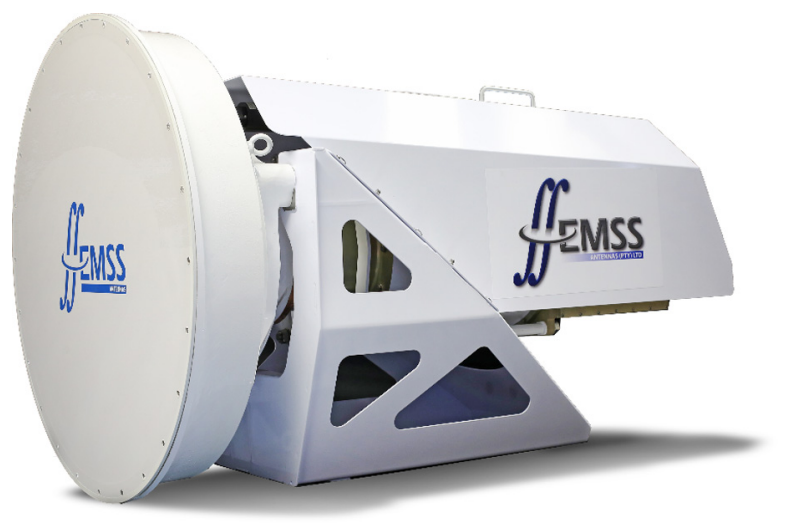

Fig. 1 The L-band receiver front-end of the SKA radio telescope. The horn's diameter is $780 \mathrm{~mm}$ and the receiver's total length is $1.24 \mathrm{~m}$.

bandwidth). Over this frequency range, the antenna temperature is about $8 \mathrm{~K}$ to $6 \mathrm{~K}$ (lowest to highest frequency) with the reflector system's main beam pointing to zenith, and increases by about $1 \mathrm{~K}$ while tipping to $60 \mathrm{deg}$ from zenith. Such a low antenna temperature dictates a low overall receiver temperature. The front-end signal chain components are in a vacuum vessel and cryogenically cooled with a Gifford-McMahon cooler to reduce the system's receiver noise temperature.

According to a recent survey of front-end receivers for radio astronomy, the best L-band receiver has a receiver noise temperature of around $8.5 \mathrm{~K},{ }^{2}$ and L-band system temperatures are above $18 \mathrm{~K}^{3-6}$ These systems are generally of narrower bandwidth-generally allowing lower noise temperatures - than the one presented in this paper.

In this paper, we describe the design of the critical components in the SKA L-band receiver front-end's signal chain (shown in Fig. 1), as well as its measured performance using a bespoke measurement setup. This system has a measured receiver temperature below $6.5 \mathrm{~K}$ and an expected system temperature of below $14 \mathrm{~K}$ when mounted on the SKA reflector system and pointing to zenith. Design details for some of the components, whether for SKA or other radio telescopes, have been presented at conferences. ${ }^{7-11}$ These details are not repeated here; the focus of this paper is on a system level.

Section 2 summarizes the receiver front-end's architecture. The reflector system and the feed horn design with some predicted performance results are given in Sec. 3. The design of the orthogonal mode transducer (OMT) and integrated directional coupler are described in Sec. 4. Section 5 presents the measured performance of the entire receiver front-end system, and Sec. 6 is the conclusion.

\section{Receiver Front-End architecture}

Figure 2 shows the main components of the receiver front-end. These consist of a wide flareangle axially corrugated conical horn, a compact OMT with an integrated symmetric directional coupler for calibration noise injection, two LNAs and second-stage amplifiers, and interconnecting coaxial cables.

The front-end signal chain components are in a vacuum vessel and cryogenically cooled with a Gifford-McMahon cooler to achieve the required low system noise temperature. The cold head has a variable speed drive that allows some control over the LNA's physical temperature. A typical operational temperature for the LNA is around $15 \mathrm{~K}$, and for the OMT and directional coupler assembly around $70 \mathrm{~K}$. The system is locally fail-safe, but all control and monitoring are done remotely over optical fiber. The SKA radio frequency interference (RFI) requirement limits the total radiated power between $300 \mathrm{MHz}$ and $2 \mathrm{GHz}$ to about $-195 \mathrm{dBm} / \mathrm{Hz}$.

The first two components of the receiver chain are the horn and the OMT, as shown in Fig. 3. The wide flare-angle axially corrugated conical horn, with its three corrugation rings, has two steps in the circular waveguide for improving the reflection coefficient of the $\mathrm{TE}_{11}$ mode close to

J. Astron. Telesc. Instrum. Syst. $\quad$ 011002-2 Jan-Mar 2022 • Vol. 8(1) 


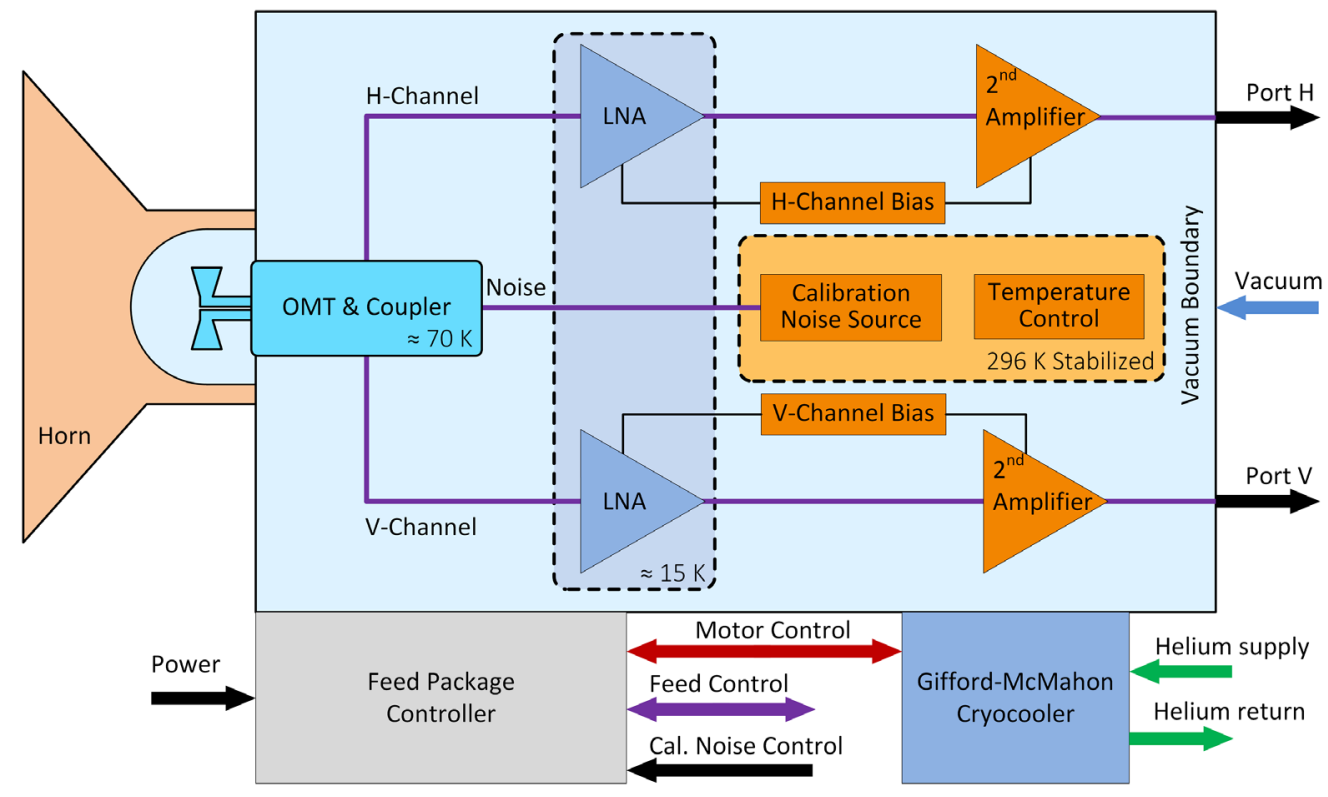

Fig. 2 An architectural overview of the SKA L-band front-end.

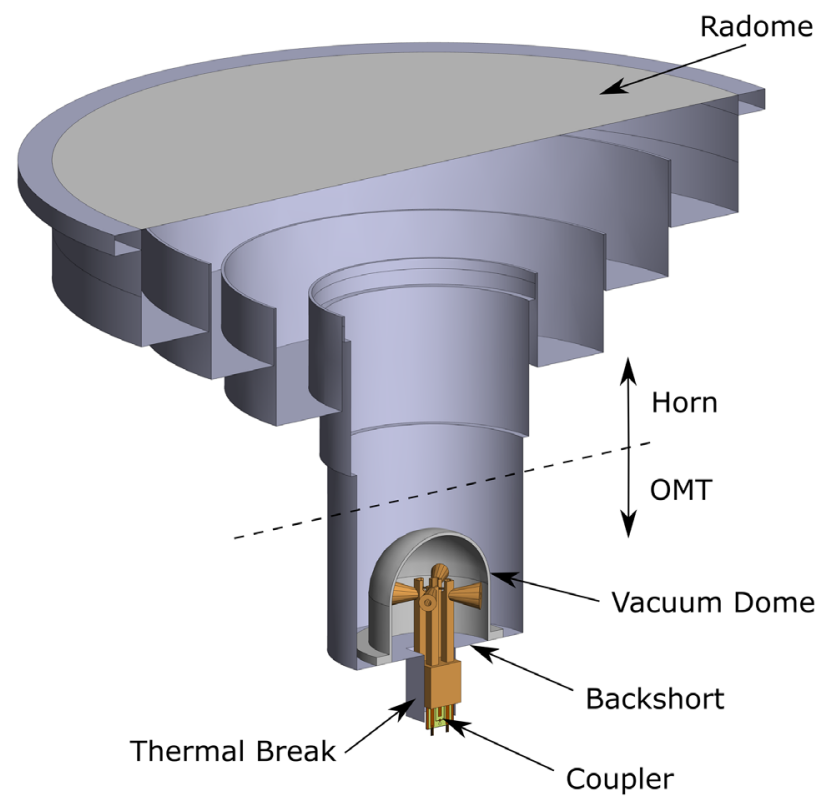

Fig. 3 The axially corrugated horn and OMT. Also shown is the environmental radome, the vacuum dome, the symmetric directional coupler, and the thermal break.

its cutoff frequency. ${ }^{7}$ An environmental radome made of fiberglass fibers and a polytetrafluoroethylene matrix protects the inside of the horn from the elements. The OMT is composed of two orthogonal dipoles placed in the circular waveguide, approximately a quarter-wavelength (at the center frequency) from a fixed backshort (see Fig. 3). It converts the balanced dipole excitations to unbalanced coaxial transmission lines using baluns, ${ }^{12}$ which are integrated into the dipole feeding structure as presented in Ref. 13. The resulting OMT structure, made from oxygen-free copper, is mechanically robust and very compact, implying low loss. ${ }^{8}$ A conceptually similar design was published in Ref. 14, where instead of a dipole structure, two probes from the sidewalls of the circular waveguide connect to a hybrid power combiner with coaxial cables. However, the design in Ref. 14 works over a narrower frequency band, and the cables and power combiner introduce extra loss. 
Also shown in Fig. 3 are the vacuum dome and the thermal break. The vacuum dome is manufactured from high-density polyethylene, and forms a barrier between the vacuum vessel and the environment, and through which the EM energy enters the OMT. A thermal break between the warm (environmental temperature) horn and circular waveguide feeding the horn, and the cold dipole and balun structure of the OMT is realized by two square coaxial conductors separated by a $1-\mathrm{mm}$ vacuum gap. See Sec. 4.2 for further details. The noise coupler is integrated into the thermal break section, and as such, does not introduce additional loss given that the thermal break has to be present.

A short coaxial cable connects the coupler to the LNA. This cable has the contradictory requirements of ensuring sufficient thermal isolation between the OMT/coupler and the LNA, and minimizing its loss contribution to the system noise temperature. Furthermore, the cable provides some mechanical decoupling between the LNA mounted on the second-stage of the Gifford-McMahon cooler, and the OMT, which is fixed by G10 brackets to the waveguide and cryostat body. (G10 is a fiberglass material that has low thermal conductive properties.) The expected noise contribution of this cable is approximately linear from $0.6 \mathrm{~K}$ to $0.9 \mathrm{~K}$ over the frequency band. The other interconnecting coaxial cables are located after the first-stage amplification and thus contribute little to the system noise. The cable between the LNA and the second-stage amplifier provides thermal isolation between the cold LNA and the room-temperature second-stage amplifier. An SMA to N-type feed-through bulkhead connector penetrates the vacuum chamber.

The LNA is the first active component in the signal chain and is responsible for determining the overall receiver noise temperature. Cables and matching/gain level-compensating attenuators after it have little impact on the system noise. If the LNA gain increases, so does its bias voltage and, consequently, the power and thermal heating; this leads to higher physical and noise temperatures. Typically, the LNA gain varies between 39 and $42 \mathrm{~dB}$ and its noise temperature is just below $2 \mathrm{~K}$. The second-stage amplifier makes up the rest of the gain to bring the overall receiver front-end gain to the required $56 \mathrm{~dB}$ level. This stage also equalizes any frequency-dependent gain and loss that the OMT, coupler, LNAs, and interconnecting cables may introduce.

\section{Reflector System and Horn}

The SKA reflector system is a shaped offset Gregorian dish with a 15-m aperture. An exhaustive parametric study that optimized feeds and optics, in turn, derived the dish parameters. ${ }^{15}$ The study aimed to obtain a dish with maximum receiving sensitivity for a given sidelobe level; wideband, octave band, and phased array feeds were considered. The selected optics has a feed subtended half-angle of $58 \mathrm{deg}$ and a 5.16-m sub-reflector chord length, including a 40-deg extension at the bottom (as measured from the secondary focus). This extension, in combination with feed-down tipping (tipping the reflector system by an angle $\theta_{\mathrm{p}}$ from zenith in the plane of symmetry in the sub-reflector's direction), reduces the noise contribution from the ground and allows increased illumination efficiency. ${ }^{16,17}$

The horn, as shown in Fig. 3, has the following design parameters: three parameters per corrugation (the corrugation depth, the corrugation width, and the offset to the next corrugation), and four parameters defining the matching steps (the length between the horn aperture and the first step, the length between the steps, and the offset of each step). Initially, the parameters of each corrugation were kept the same, and a global optimizer was applied. These parameters for the horn then served as the input to a local optimizer.

In each iteration of the optimizer, the EM analysis followed a two-step process. First, the horn was analyzed using a method of moments (MOM) solver, namely, "Feldberechnung für körper mit beliebiger oberfläche" (FEKO), ${ }^{18}$ to determine the circular waveguide $\mathrm{TE}_{11}$ mode reflection coefficient into free space and the horn's radiation pattern. The effect of the sub-reflector on the horn's reflection coefficient is a ripple superimposed on the free space reflection coefficient. Feed optimization cannot minimize this effect. The feed's phase center was determined from the horn's radiation pattern. ${ }^{19}$ Second, the feed (using the spherical mode expansion coefficients), positioned at the focus, was used to illuminate the reflector system using the physical optics (PO) and physical theory of diffraction method of "general reflector antenna software

J. Astron. Telesc. Instrum. Syst. 011002-4 Jan-Mar 2022 • Vol. 8(1) 
package" (GRASP) ${ }^{20}$ to determine the reflector's far-field. An ideal OMT was assumed in the optimization, i.e., using symmetry and feeding the horn with a $\mathrm{TE}_{11}$ mode.

The objective function comprised a weighted sum of the following goals over the frequency band: maximize receiving sensitivity, minimize $\mathrm{TE}_{11}$ mode reflection coefficient, limit maximum sidelobe level, limit maximum cross-polarization (IXR) level, and limit drop-off of efficiency at the highest frequency. The specific weightings were adjusted during optimization to take into account the order of design preference: maximize sensitivity, minimize variation in sensitivity, minimize far-out sidelobes, minimize larger far-out sidelobe extent, and minimize near-in sidelobes.

After the full optimization, the horn and dish were analyzed in both GRASP and FEKO. FEKO used the multilevel fast multipole method, which takes the full interaction between the horn, the sub-reflector, and the main reflector into account. Figure 4 shows the input reflection coefficient of the horn radiating into free space. It is well below $-25 \mathrm{~dB}$ over most of the band but climbs to around $-21 \mathrm{~dB}$ at the lower frequencies because of the relatively small electrical size of the circular waveguide feeding the horn. The OMT optimization needs to take into account the horn's complex reflection coefficient, to achieve a good match at the coaxial ports of the OMT.

Figure 5 is a plot of the aperture efficiency $\eta_{a}$. It is worth noting that the horn was primarily optimized for receiving sensitivity, which implies that the efficiency is not necessarily maximized for the given reflector system. The resultant first and second sidelobe levels are around -18 and $-26 \mathrm{~dB}$, respectively. The intrinsic IXR levels for the -1 and $-3 \mathrm{~dB}$ contours of the main beam are generally greater than 45 and $35 \mathrm{~dB}$, respectively.

With a sky brightness temperature model as given in Ref. 21, the receiving sensitivity per SKA dish for the vertical polarization was predicted and is shown in Fig. 6 over both frequency and tipping angle, where $\theta_{\mathrm{p}}=0 \mathrm{deg}$ is zenith. The horizontal polarization is equivalent. The analyses used a receiver temperature that varies linearly from $6 \mathrm{~K}$ to $7.5 \mathrm{~K}$ over the frequency band (including $1 \mathrm{~K}$ noise for the back-end; see Sec. 5). A very flat sensitivity is observed over frequency and tipping angles to around $60 \mathrm{deg}$. The average sensitivity for $\theta_{\mathrm{p}} \in\left[\begin{array}{lll}0 \mathrm{deg}, 70 \mathrm{deg}\end{array}\right]$ over the entire frequency band is $11.3 \mathrm{~m}^{2} / \mathrm{K}$, or equivalently $T_{\text {sys }} / \eta_{\mathrm{a}}=15.6 \mathrm{~K}$.

\section{OMT and Calibration Noise Injection}

Figure 7 shows a detailed view of the physical structure of the OMT. The structure comprises two orthogonal sets of input coaxial transmission lines, dipole feeders, open-circuited coaxial transmission lines, and short-circuited twin-wire transmission lines realized by the outside conductor

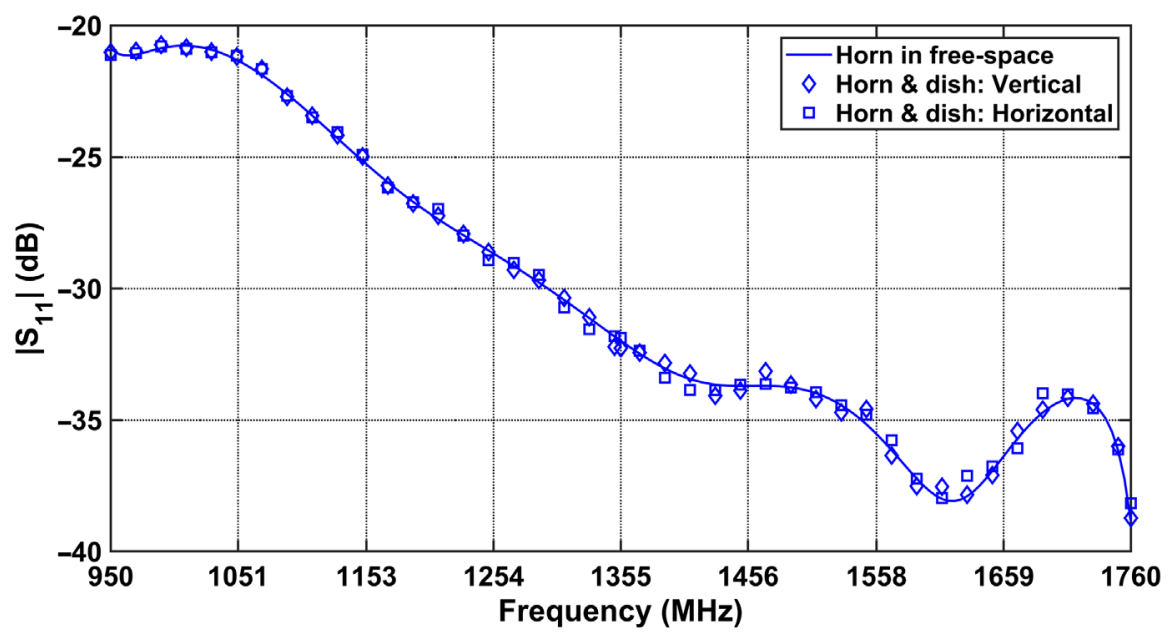

Fig. 4 The circular waveguide TE11 mode reflection coefficient of the optimized horn calculated with FEKO for the horn radiating into free space, and when illuminating the reflectors for both polarizations. 


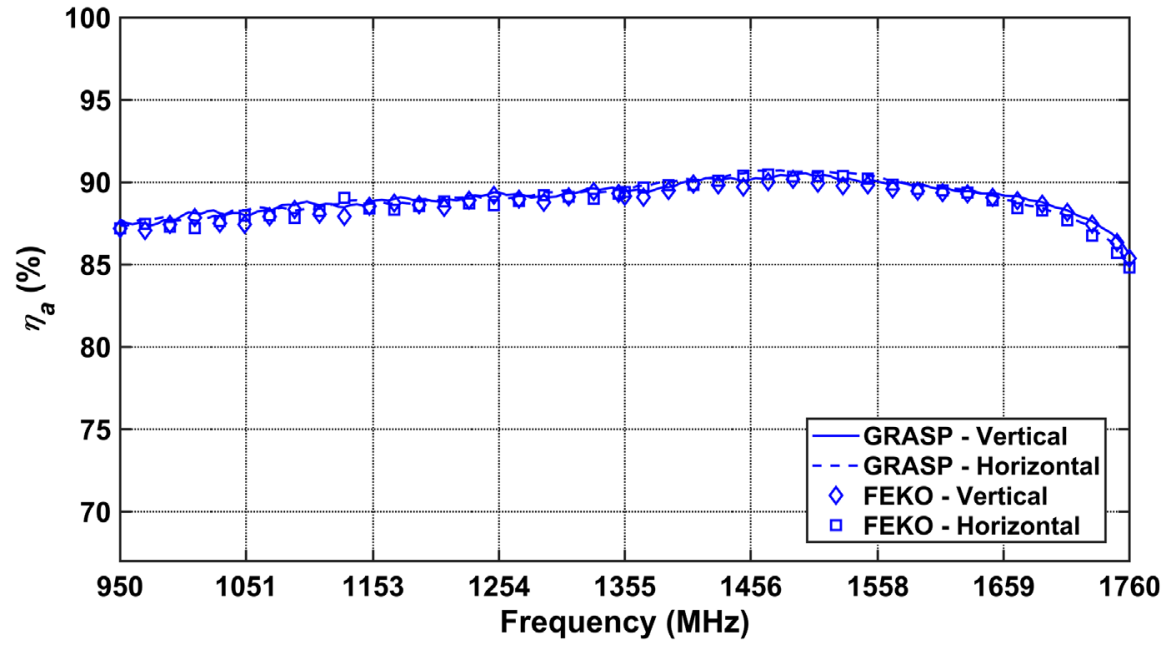

Fig. 5 The aperture efficiency of the optimized reflector-horn combination calculated with GRASP and FEKO for both polarizations.

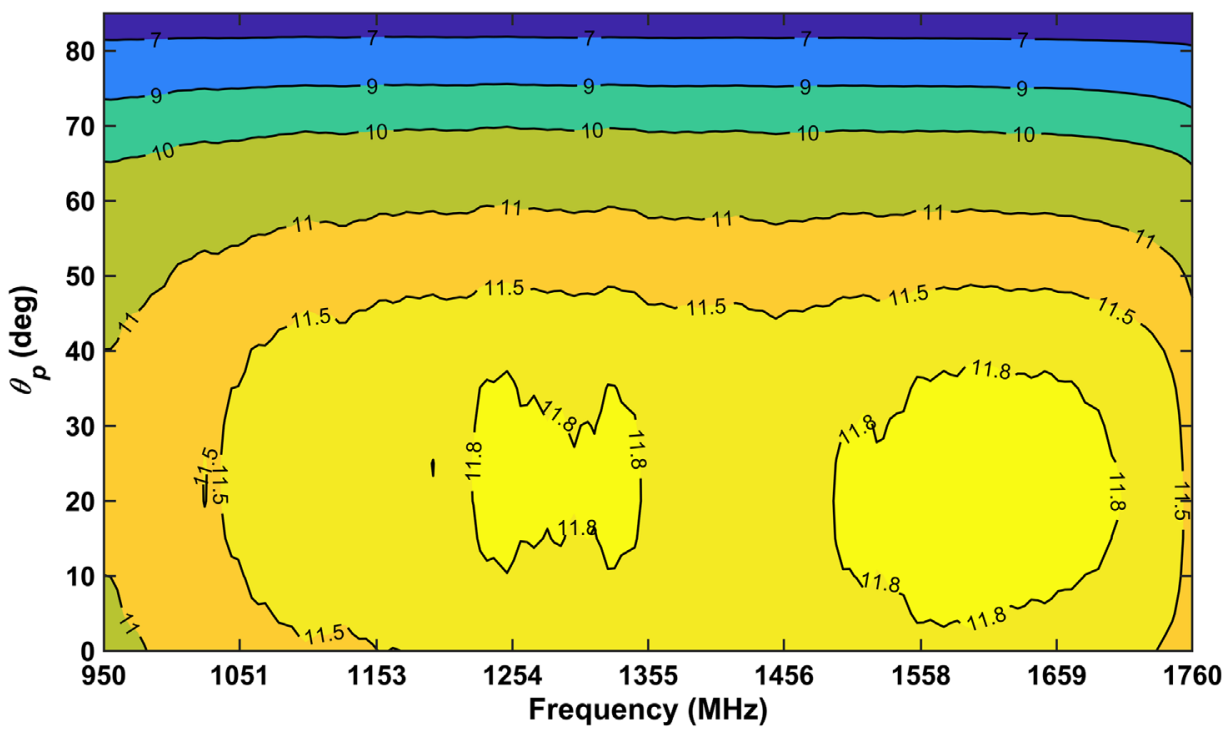

Fig. 6 The receiving sensitivity $\left(\mathrm{m}^{2} / \mathrm{K}\right)$ for the vertically polarized signal over all pointing angles $\theta_{\mathrm{p}}$ and frequency calculated from patterns determined with GRASP.

of the two coaxial transmission lines. The orthogonal dipoles are conical to reduce the variance of the dipole impedance over the frequency band. The chosen circular waveguide diameter ensures that the next higher symmetric mode $\left(\mathrm{TM}_{11}\right)$ is just below cutoff at the maximum frequency. Thus, only the fundamental $\left(\mathrm{TE}_{11}\right)$ and two asymmetric $\left(\mathrm{TM}_{01}\right.$ and $\left.\mathrm{TE}_{21}\right)$ modes, which for a symmetrical feeding structure will not be excited, can propagate in the frequency band. Due to the unsymmetrical OMT feeding structure and machining tolerances, a small amount of coupling to the asymmetric modes does exist and causes slight asymmetry in the horn's radiation pattern.

\subsection{OMT}

The feed wires between the two arms of the orthogonal dipole pairs must be physically separated. The separation determines the coupling between them, and was chosen so that the theoretical isolation between the two polarizations is above $50 \mathrm{~dB}$. Thus, the embedded all-pass filter design for the two polarizations is not identical, implying an asymmetric OMT 


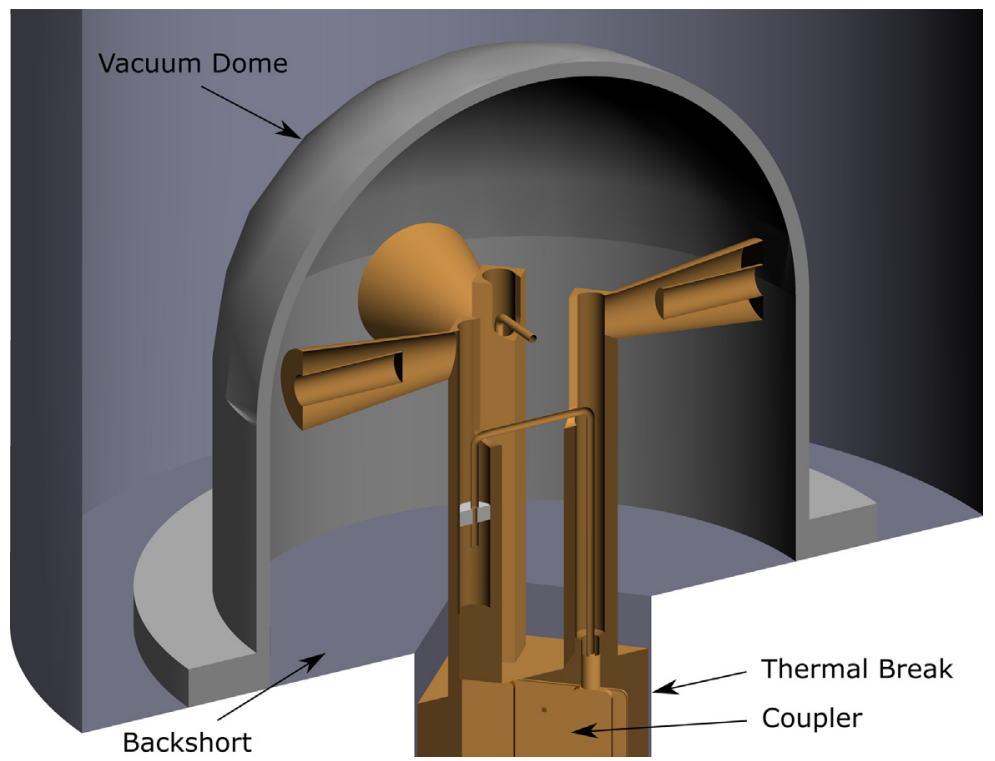

Fig. 7 A detailed view of the OMT with a section-cut through one of the linear polarizations.

structure. The short on the twin-wire transmission lines should then ideally be at unique positions, which can be done by adding a vertical plate (on the backshort side) between the conductors of one of the twin-wire lines. ${ }^{8}$ As this plate complicates the manufacturing process, it is omitted from the OMT design. This has a slight impact on optimized performance. As the lengths of the short-circuited transmission lines are longer than the length from the dipole feeders to the backshort, the twin-wire line shorts are behind the backshort and form part of the thermal break.

The OMT has several design variables, which include the parameters that affect the input and open-circuit coaxial transmission lines, the short-circuit twin-wire transmission line and the dipole radiators. Even with some parameters chosen as fixed values, the OMT has 18 design parameters. The wide frequency band and low required reflection coefficients dictate the use of full-wave EM analyses to account for all the EM interactions within the feed (i.e., horn and OMT), not only for the final design, but also through the design process. The MOM analysis time is long and, given the large design parameter space, prohibits using any direct optimization method. In light of this, the optimization was done by decomposing the full model into several high-fidelity and (where possible) low-fidelity models. ${ }^{9}$

The predicted and measured results of the input reflection coefficients and the isolation between the orthogonal ports of a prototype are given in Fig. 8. In the analyses, port $\mathrm{H}$ (horizontal) corresponds with the structure that has the dipole feeder below that of port V (vertical). For the final design, the OMT was scaled to compensate for the thermal shrinkage that occurs when the OMT is cooled to its operating temperature inside the cryostat.

\subsection{Thermal Break}

The thermal break (see Fig. 9) is essentially a coaxial waveguide with a very low characteristic impedance $(2.6 \Omega)$ for the TEM mode and with all higher-order modes evanescent in the frequency band $\left(\mathrm{TE}_{11}\right.$ 's cutoff frequency, for instance, is at $\left.2.06 \mathrm{GHz}\right)$. The predicted EM leakage through the thermal break is below $-60 \mathrm{~dB}$ across the frequency band.

Although the thermal break offers high attenuation, the signal path also has a large gain $(\sim 56 \mathrm{~dB})$. As the cryostat body is electrically large, it supports several resonant modes-any coupling from the output of the amplifier stages to modes in the cryostat body could thus potentially reach the OMT waveguide and result in increased coupling between output ports. A radial quarter-wavelength choke was therefore added to the thermal break and provided an additional $20 \mathrm{~dB}$ attenuation to the TEM mode. 


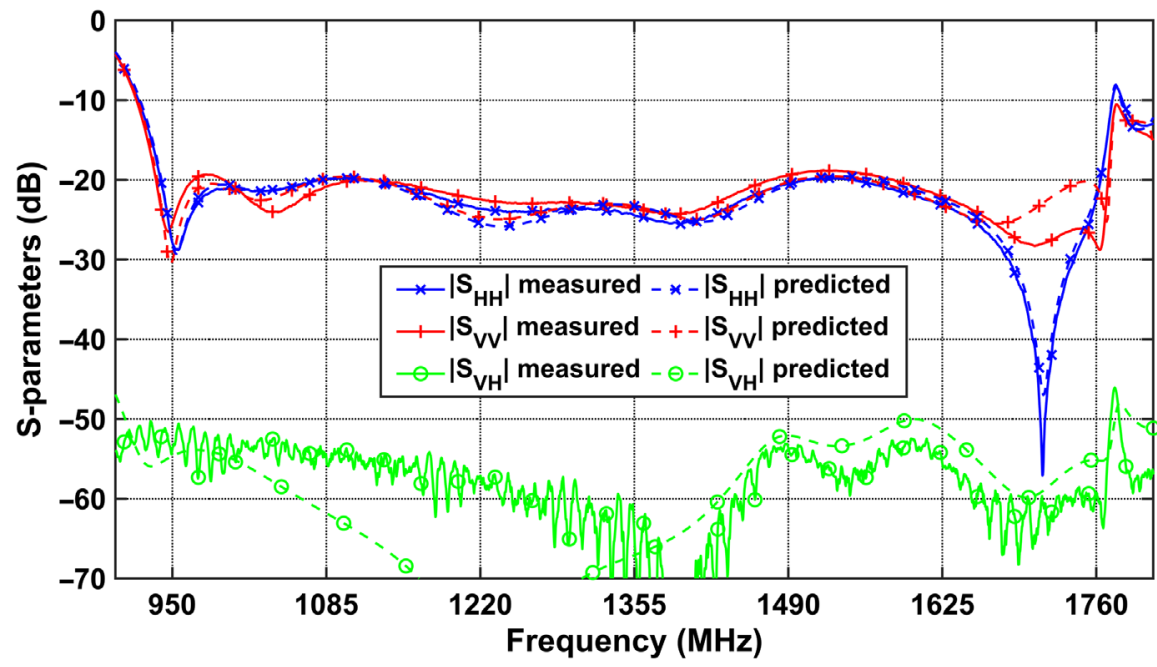

Fig. 8 The predicted and measured reflection coefficients of the OMT and horn, and the coupling between the OMT channels.

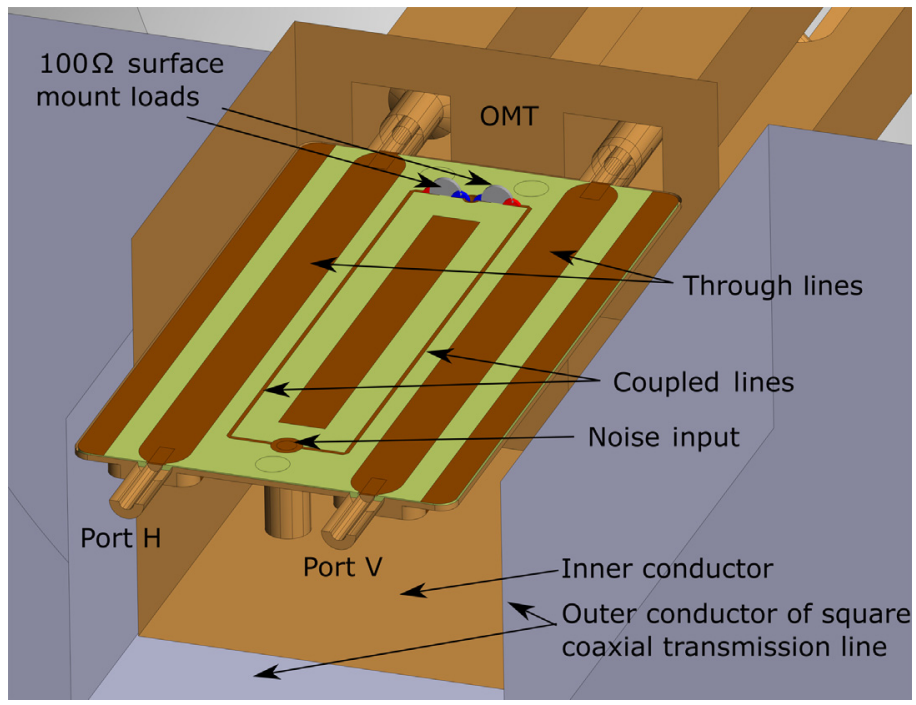

Fig. 9 A model of the symmetric directional coupler and thermal break.

\subsection{Directional Coupler}

Calibrating the signal path for radio astronomy observations relies on injecting a well-known noise signal (generated by a noise diode). This is typically at a level of about $10 \%$ of the system temperature. Using a symmetric coupler, as in Ref. 8, requires only one noise source and allows for full phase calibration between the two channels. The coupler is integrated as part of the thermal break directly after the OMT.

The SKA requirement is for the power spectral density of the injected calibration signal to be between $5 \%$ and $13 \%$ of the system temperature when pointing at cold sky at an elevation of $30^{\circ}$, which implies an injection noise between $0.7 \mathrm{~K}$ and $1.9 \mathrm{~K}$ approximately. When the noise source, which is at room temperature, is switched off, its noise coupling into the signal path has to be very low. A coupling level of $35 \mathrm{~dB}$ kept this level low while enabling the use of noise diodes with fairly low excess noise ratios.

Figure 9 shows a FEKO model of the single section quarter-wavelength directional coupler design. The through transmission lines are suspended striplines, and the substrate is RT/Duroid 5870 with a thickness of $0.508 \mathrm{~mm}$. The substrate has thermal expansion coefficients similar to 


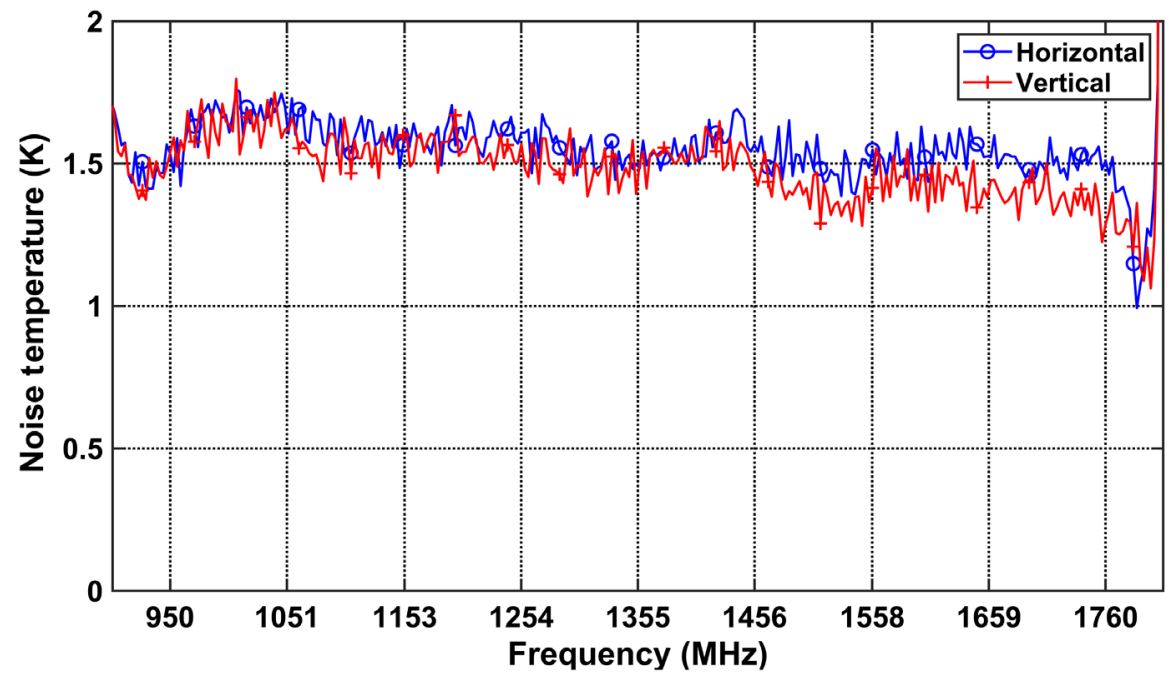

Fig. 10 The measured noise contribution from noise injected into the calibration port.

that of copper and has low outgassing properties. For the through-lines, the substrate thickness is minimal relative to the ground plane spacing. As a result, most of the electric field lines are in free space, thus minimizing the coupler's contribution to the receiver noise. The coupled lines are $100 \Omega$ covered microstrip transmission lines that, on the one side (coupler input), are fed with a single stub to facilitate symmetric coupling to both through-lines, with the other side (isolation ports) terminated in $100 \Omega$ loads. The lines are in parallel so that the noise port input impedance is $50 \Omega$.

Optimization of the design was done in FEKO. The optimization was expedited, as parameters that affect the different performance levels are easily identified and have effects that are reasonably easy to predict. For instance, the distance between the coupled lines varies the mean coupling level. It is worth noting that the distance between the through-lines is fixed during the OMT design. The coupler is positioned symmetrically between the two through-lines, ensuring symmetric coupling. The impedance matching of the through-lines does not change significantly with modifications to the coupler printed circuit board and has been optimized for a match lower than $-30 \mathrm{~dB}$.

The quarter-wavelength coupler has a $1 \mathrm{~dB}$ coupling level variation over the SKA band 2 frequency band. This variation (and to a lesser degree, the cable connecting the noise source to the coupler, as well as the noise diode variations over frequency) was compensated for by a shaping circuit after the noise diode.

The measured noise contribution from noise injected into the calibration port into the signal chain is given in Fig. 10.

\section{Low-Noise Amplifier}

The system has two cryogenic LNAs from the Herzberg Astronomy and Astrophysics Research Center. ${ }^{10}$ The single-ended LNA design has been developed on GaAs High-Electron-Mobility Transistors (GaAs HEMTs) in three-stage amplification. GaAs HEMT is a mature III/V semiconductor technology. The transistor performance is quite stable and repeatable, which is essential for mass production of LNAs. As the first amplifier in the signal chain, the noise level and input return loss of this LNA would affect the receiver sensitivity and efficiency. The noise performance of the LNAs depends on the noise matching, which is correlated with their input return losses. The design challenge is to achieve the lowest possible noise and, simultaneously, a good input return loss to minimize the reflection (loss) of collected signals back to the sky.

The LNA body is made from tellurium copper and gold-plated for cryogenic cooling purposes. All of the circuit components should be vacuum-compatible materials. They are directly mounted on a gold-plated chassis surface to achieve the best thermal conductivity and to ensure 
that the transistors are cooled efficiently at a stable temperature during operation. Unlike roomtemperature LNAs, which are typically hermetically sealed, the cryogenic LNA chassis has a vent hole to allow for air to escape before cooling it. Any trapped air inside the chassis would freeze at $15 \mathrm{~K}$ and could cause damage to the circuit. The circuit layout is also critical to achieving reliable performance under cryogenic temperature and thermal cycling as thermal contraction and expansion can lead to thermal stress on components. This mechanical stress on components and their connections must be prevented in the circuit layout.

The LNAs were measured at a physical temperature of $15 \mathrm{~K}$. Their noise temperatures, as measured with the cooled attenuator method, are plotted in Fig. 11. The noise is mostly below $1.5 \mathrm{~K}$. Figure 12 shows the measured gains of the LNAs. It is worth noting that the gain is not tightly controlled as the design is optimized toward noise and stability. The typical input and output return losses exceed $10 \mathrm{~dB}$, as shown in Fig. 13. These LNAs are unconditionally stable when connected to the OMT and feed horn at $15 \mathrm{~K}$.

\section{Measurements of the Front-End System}

Traditionally, noise is measured with the Y-factor method that requires the feed to point at a hot and a cold load. ${ }^{22}$ These loads would be radiation-absorbent material (RAM) at room

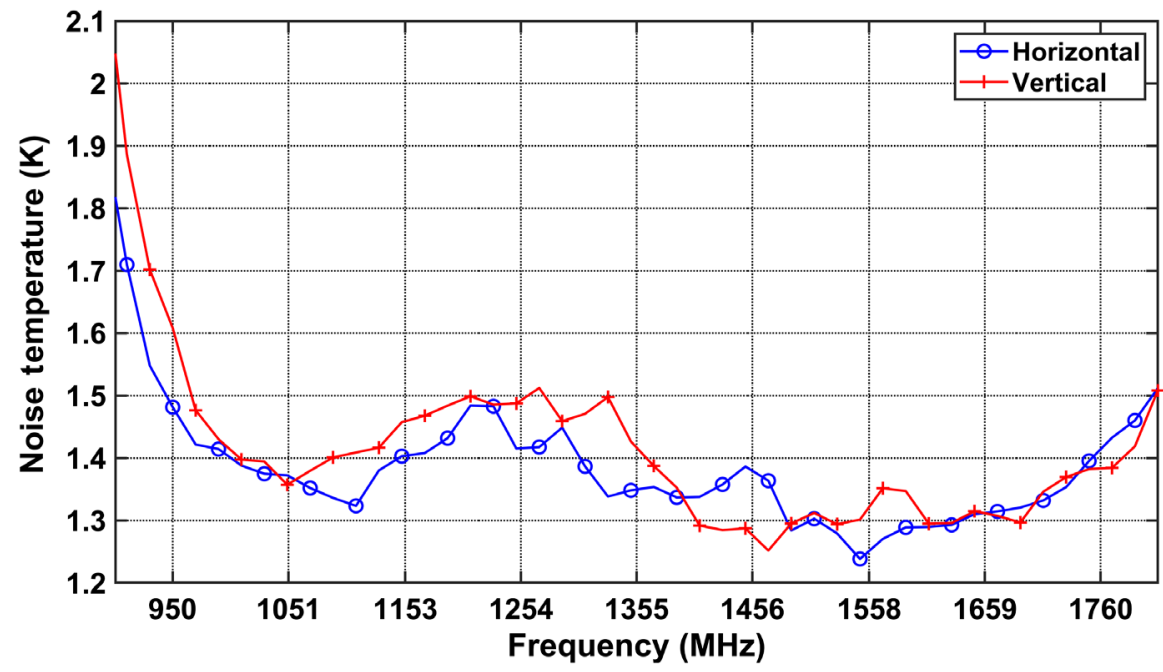

Fig. 11 The measured noise temperature of the LNAs at $15 \mathrm{~K}$.

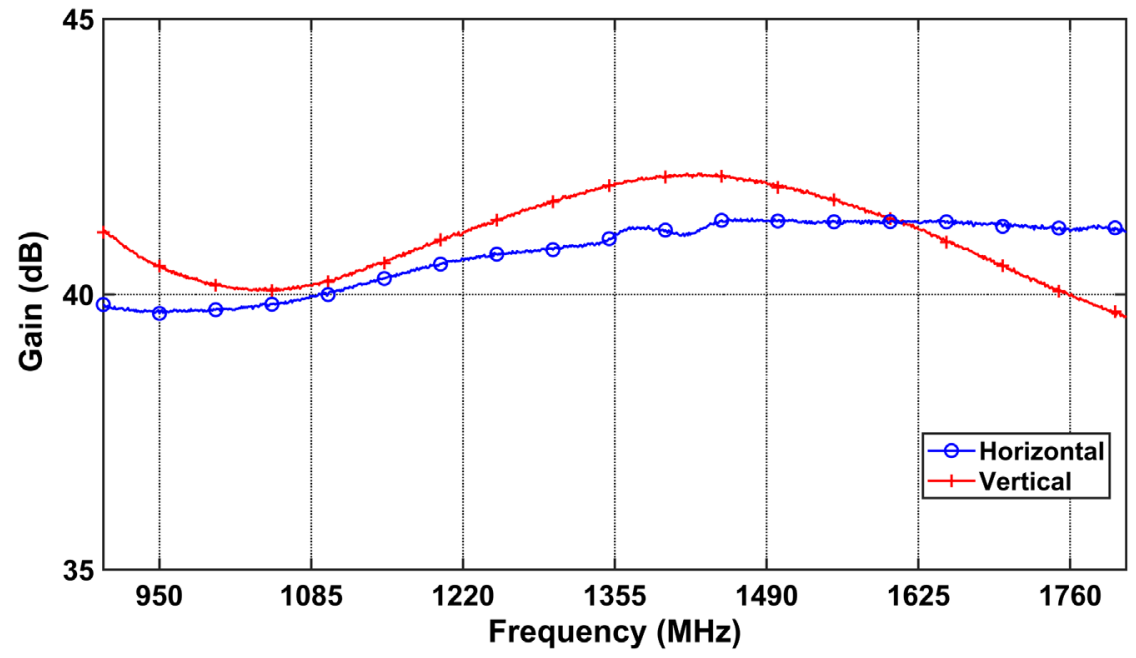

Fig. 12 The measured gains of the LNAs at $15 \mathrm{~K}$. 


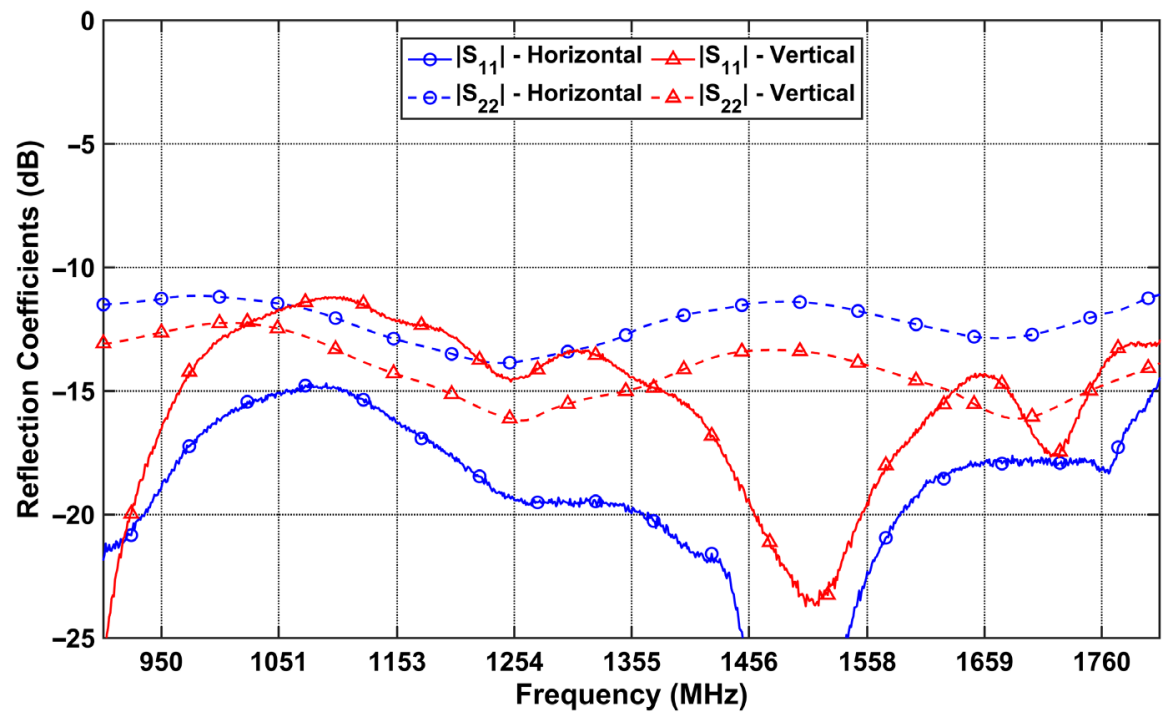

Fig. 13 The LNA's reflection coefficients measured at $15 \mathrm{~K}$.

temperature (hot load) or soaked in liquid nitrogen (cold load). The load would have to be larger than the horn and thus require more liquid nitrogen than is safe to handle in an EM laboratory. An alternative is to point the feed at cold sky for the cold load; this measurement would have to be done in a radio-quiet area so that RFI does not severely perturb the results. Both these techniques are time-consuming and impractical for manufacturing done in a populated area.

For non-radiating components, the cold attenuator method can be used, which makes use of a calibrated noise source connected to an attenuator at a low physical temperature. The hot load is provided by switching the noise source on. With the noise source off, the attenuator provides a cold load that is equal to its physical temperature. A significant advantage of this method is that the input noise temperature can be changed very rapidly, compared with cooling a physical load, thus minimizing drift between measurements. This method requires a coaxial-to-waveguide transition to connect to the SKA front-end with its horn, which has insignificant noise contribution, removed. Another advantage of having a coaxial-to-waveguide transition is that known signals can be injected into the receiver front-end for its characterization.

As the circular waveguide between the OMT and horn is non-standard and supports multiple modes, a bespoke single-mode transducer (SMT) design is required. An SMT (at room temperature) allows the receiver front-end's S-parameters to be measured with a vector network analyzer. A cryogenically cooled (to reduce parasitic noise) SMT and an attenuator, which is precisely maintained at $18 \mathrm{~K}$, as well as a calibrated noise source controlled at $28^{\circ} \mathrm{C}$, are used to measure the system's noise temperature with a spectrum analyzer.

The properties of all components (both noise due to losses/physical temperature and S-parameters) between the calibrated noise source and the input of the receiver front-end need to be characterized accurately, using measurements to ensure that their influence can be deembedded from the measurement results. Some of these components are at cryogenic temperatures, with large temperature gradients, making the characterization difficult.

A thru-reflect-line (TRL) calibration method was used to characterize the SMT. ${ }^{23}$ This method is limited in frequency range but does not require a good dissipative load, which may be difficult to realize at low frequencies. Furthermore, the assumption is made that only a single mode exists at the OMT-SMT interface, and the coupling to other modes is low. Despite these drawbacks, the obtained calibration compares well to simulated results.

\subsection{SMT Design}

The primary function of the SMT is to act as a low-loss through-line for the fundamental $\mathrm{TE}_{11}$ mode being tested, with reflections in the same order as when the OMT is used with the horn. 
The OMT, however, is not matched to waveguide, having been matched to the horn, and thus requires the SMT to be optimized for a specific OMT design. As the OMT is not symmetric, the SMT optimization needs to be a compromise for both ports. The circular waveguide supports three propagating modes, namely, the $\mathrm{TE}_{11}, \mathrm{TM}_{01}$, and $\mathrm{TE}_{21}$ modes (where $\mathrm{TE}_{11}$ and $\mathrm{TE}_{21}$ also have orthogonal versions). The higher-order $\mathrm{TE}_{21}$ and $\mathrm{TM}_{01}$ modes are typically not excited in the OMT waveguide, and if excited due to manufacturing tolerances, their modal content compared with the $\mathrm{TE}_{11}$ fundamental mode will be very low in the OMT on its own.

Connecting two OMTs back-to-back results in a high-Q resonant cavity for the higher-order modes, as the OMTs exhibit very low loss and reflect the higher-order modes almost perfectly. Any discontinuity or misalignment in the structure will excite higher-order modes and cause high-Q resonances. These resonances then cause loss, which results in sharp increases in the noise measurements. The higher-order modes thus need to be attenuated or absorbed into loads by the SMT. The method used to absorb the higher-order modes must not have a significant impact on the fundamental mode and should not couple noise into the receiver front-end under test.

By observing the field distributions of the various modes in the waveguide, methods to absorb the modes can be devised. (Refer to the SMT design shown in Fig. 14 for the explanation below.) The dominant $\mathrm{TE}_{11}$ mode is converted by a center-fed dipole in the waveguide, as is done in the OMT. The $\mathrm{TE}_{21}$ modes can be absorbed by monopoles in the sidewalls of the OMT as well as by curved dipoles arranged along the electric fields, off-center to the waveguide. By their symmetry, they will not affect the $\mathrm{TE}_{11}$ mode as the electric field is directed in opposite directions on either side of the dipoles. The unwanted orthogonal $\mathrm{TE}_{11}$ mode also has to be absorbed. This requires a set of monopoles in the center of the waveguide as the monopoles in the sidewalls used to absorb $\mathrm{TE}_{21}$ are not sufficient for this across the frequency band. Lastly, the $\mathrm{TM}_{01}$ mode has an electric field directed along the waveguide axis and can be absorbed using monopoles that extend from the backshort upward toward the OMT. Note that the dipoles and feed structure cause the electric field distribution to change significantly, especially for the $\mathrm{TM}_{01}$ mode (where the electric field is directed along the feed lines).

Each of the elements used to absorb a mode was optimized in turn. The simulated reflection coefficient for such an optimized load is shown in Fig. 15. The peak values at $1153 \mathrm{MHz}$ and $1465 \mathrm{MHz}$ are the $\mathrm{TM}_{01}$ and $\mathrm{TE}_{21}$ mode cutoff frequencies, respectively. Below these frequencies, the respective modes do not propagate. Above these frequencies, it can be seen that the modes are absorbed to varying degrees. All the loads used to terminate the absorbing elements are cryogenically cooled to reduce their noise contribution.

The $\mathrm{TM}_{01}$ mode is difficult to absorb without affecting the fundamental mode. However, simulations indicate that the absorption offered by the SMT should be sufficient to reduce the quality factor of the resonant cavity significantly and prevent any sharp resonances from occurring. It should be noted that the cavity modes $\mathrm{TM}_{010}$ and $\mathrm{TE}_{210}$ (resonant modes at cutoff) do not cause resonances.

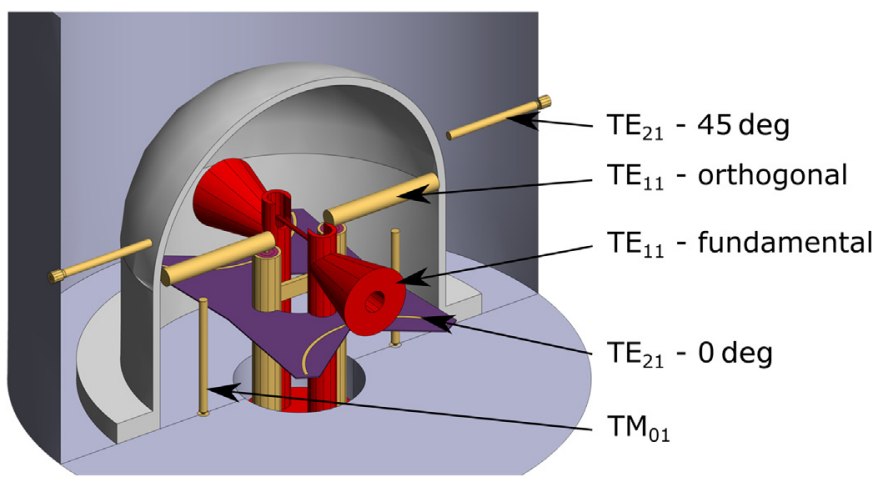

Fig. 14 The SMT used as a waveguide load and for signal injection to characterize the SKA Lband feed. 


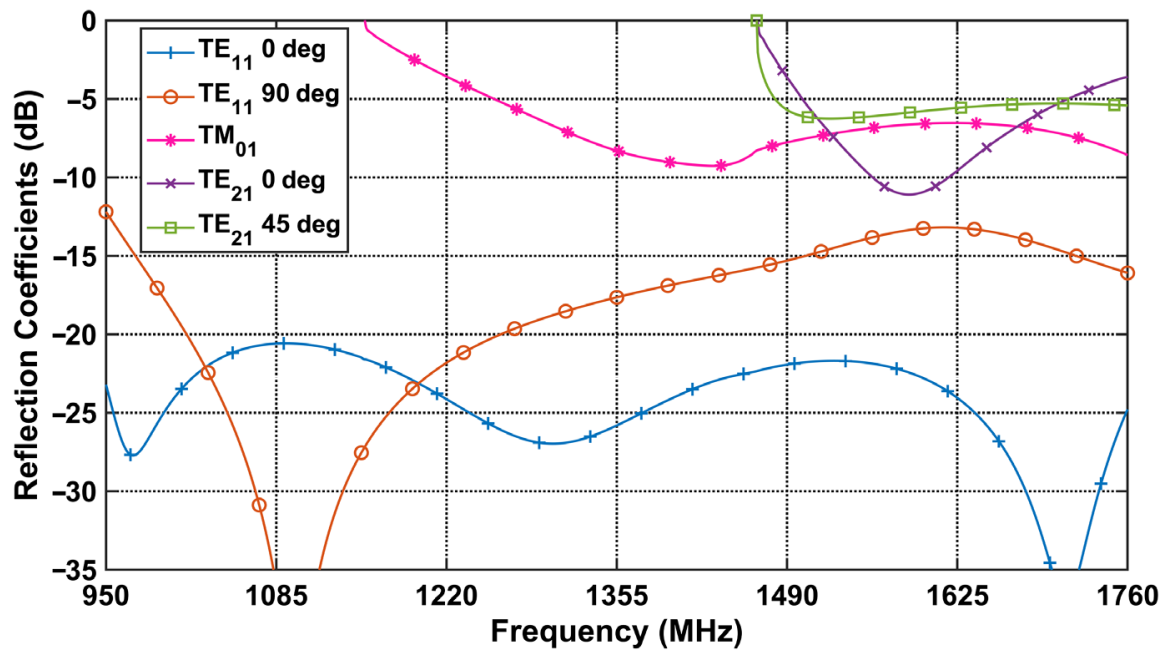

Fig. 15 Simulated reflection coefficients for the optimized SMT.

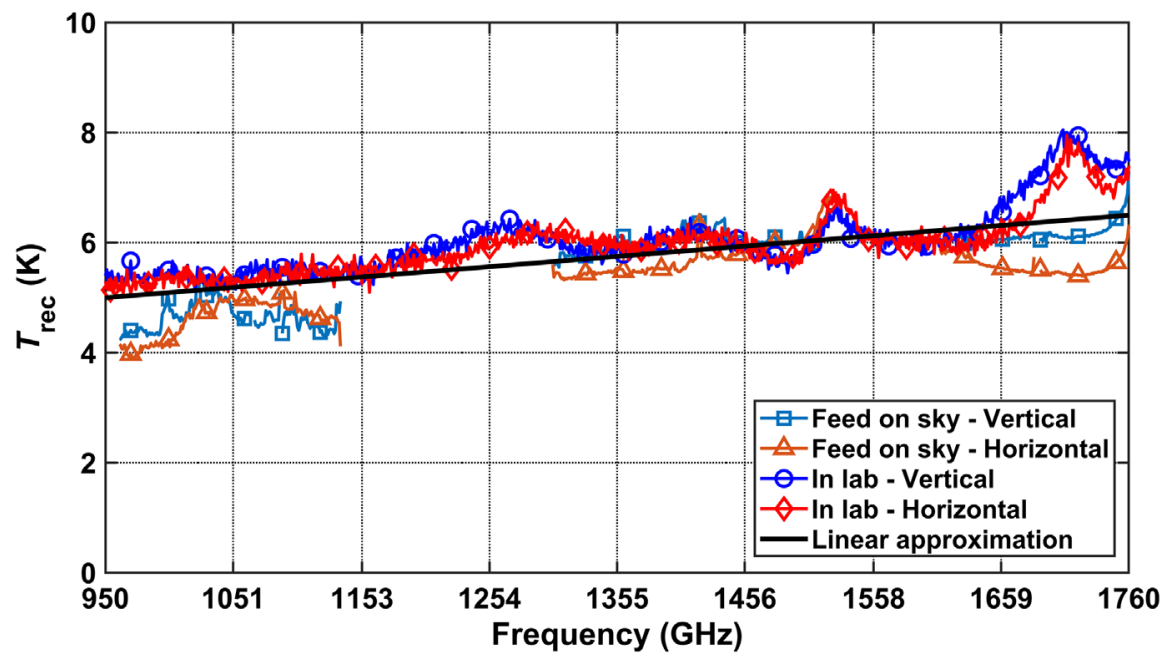

Fig. 16 The measured receiver noise temperature.

\subsection{Measurement Results}

A prototype receiver front-end was measured in a laboratory, in an RFI shielded tent with a shielding effectiveness of $80 \mathrm{~dB}$. The result is shown in Fig. 16. To verify the validity of the measurement, the system was measured using the cold sky/hot load Y-factor measurement technique at the radio-quiet Karoo site. A good correlation is observed. A linear fit of the measured data shows that the receiver temperature varies from $5 \mathrm{~K}$ to $6.5 \mathrm{~K}$ over the frequency band.

\section{Conclusion}

The design of the SKA L-band radio astronomy receiver front-end and a technique to measure this system in a laboratory with automated control and processing software were described. At the heart of the design is a compact OMT with a reflection coefficient of $-20 \mathrm{~dB}$ and a crosscoupling of less than $-50 \mathrm{~dB}$, and two LNAs with very low noise temperature. The low OMT reflection coefficient ensures low gain ripple across the frequency band and the OMT is void of resonances across its entire frequency band. A symmetric directional coupler allows injecting calibration noise using a single noise source. The system has a very wide frequency band for the technology used and a very low measured noise contribution of around $6 \mathrm{~K}$. The feed horn's 
main beam is symmetric and stable over frequency, and when combined with the SKA reflector system, produces an average receiving sensitivity over both the tipping angle up to 70 deg and the entire frequency band of $11.3 \mathrm{~m}^{2} / \mathrm{K}$, or equivalently $T_{\text {sys }} / \eta_{\mathrm{a}}=15.6 \mathrm{~K}$.

\section{References}

1. P. E. Dewdney et al., "The Square Kilometer Array," Proc. IEEE 97(8), 1482-1496 (2009).

2. P. Bolli et al., "An international survey of front-end receivers and observing performance of telescopes for radio astronomy," Publ. Astron. Soc. Pac. 131(1002), 085002 (2019).

3. R. J. Maddalena, "The performance of the GBT," https://www.gb.nrao.edu/GBT/ Performance/PlaningObservations.htm (2021).

4. "ATCA users guide," https://www.narrabri.atnf.csiro.au/observing/users_guide/html/atug .html (2018).

5. R. Nan et al., "The five-hundred-meter aperture spherical radio telescope (FAST) project," Int. J. Mod. Phys. D 20(6), 989-1024 (2011).

6. I. Prandoni et al., "The Sardinia radio telescope, from a technological project to a radio observatory," Astron. Astrophys. 608, 26 (2017).

7. R. Lehmensiek and D. I. L. de Villiers, "Wide flare angle axially corrugated conical horn design for a classical offset dual-reflector antenna," in Proc. 6th Eur. Conf. Antennas and Propag.), Prague, pp. 3292-3294 (2012).

8. R. Lehmensiek and I. P. Theron, "Compact low loss L-band orthomode transducer," in Proc. Int. Conf. Electromagn. Adv. Appl., Sydney, pp. 228-231 (2010).

9. R. Lehmensiek, "On the electromagnetic design of a horn and orthogonal mode transducer for the SKA band 2 feed with high-fidelity performance," in Proc. VII Eur. Congr. Comput. Methods Appl. Sci. and Eng., Crete (2016).

10. N. Jiang et al., "Cryogenic L-band GaAs HEMT LNA for the Square Kilometer Array," in Proc. 18th Int. Symp. Antenna Technol. Appl. Electromagn. (2018).

11. R. Lehmensiek and I. P. Theron, "Measurements of the SKA Band 2 radio astronomy receiver," in Proc. Int. Conf. Electromagn. Adv. Appl., Granada (2019).

12. N. Marchand, "Transmission-line conversion transformers," Electronics 17(12), 142-145 (1944).

13. W. K. Roberts, “A new wideband Balun," Proc. IRE 45, 1628-1631 (1957).

14. G. Valente et al, "A compact L-band orthomode transducer for radio astronomical receivers at cryogenic temperature," IEEE Trans. Microwave Theory Tech. 63(10), 3218-3227 (2015).

15. R. Lehmensiek, I. P. Theron, and D. I. L. de Villiers, "Deriving an optimum mapping function for the SKA shaped offset Gregorian reflectors," IEEE Trans. Antennas Propag. 63(11), 4658-4666 (2015).

16. R. Lehmensiek and D. I. L. de Villiers, "Sensitivity performance of the SKA offset Gregorian reflector candidates with ideal feeds," in Proc. IEEE Int. Symp. Antennas and Propag. (APSURSI), Memphis, Tennessee, pp. 1479-1480 (2014).

17. D. I. L. de Villiers and R. Lehmensiek, "Sub-reflector extensions in shaped dual reflector systems," in Proc. IEEE Int. Symp. Antennas and Propag., Memphis, Tennessee, pp. 14801482 (2014).

18. “Altair, FEKO, Suite 7.0," http://www.feko.info (2015).

19. P.-S. Kildal, "Combined E- and H-plane phase centers of antenna feeds," IEEE Trans. Antennas Propag. 31(1), 199-202 (1983).

20. "TICRA, GRASP10, Version 10.0.1, Copenhagen, Denmark," http://www.ticra.com (2012).

21. G. C. Medellín, "Antenna noise temperature calculation," in SKA Memo 95 (2007).

22. J. D. Kraus, Radio Astronomy, 2nd ed., Cygnus-Quasar, Powell, Ohio (1986).

23. G. F. Engen and C. A. Hoer, "Thru-reflect-line: An improved technique for calibrating the dual six-port automatic network analyzer," IEEE Trans. Microwave Theory Tech. 27(12), 987-993 (1979).

Robert Lehmensiek is a senior engineer and director at EMSS Antennas, and a professor (außerordentlicher) at the University of Stellenbosch. He received his BEng, MEng, and 
$\mathrm{PhD}$ degrees in electronic engineering from the University of Stellenbosch in 1992, 1995, and 2001, respectively. His research interests include reflector system and cryogenic receiver design for radio astronomy.

Ockert B. Jacobs is senior engineer at Alphawave Golf. He received his BEng and MEng degrees in electronic engineering from the University of Pretoria in 2009 and 2011, respectively. His research interests include antennas for radio astronomy, wideband antennas, and short range radar systems.

Nianhua Jiang is senior research council officer at National Research Council of Canada, and adjunct professor with Department of Electrical and Computer Engineering, University of Victoria. He received the BEng and MEng degrees in electronic engineering from University of Electronic Science and Technology of China, in 1982 and 1984, respectively, and the $\mathrm{PhD}$ in electrical and communication engineering from Tohoku University, Sendai, Japan, in 1996. His research interests include low-noise amplifiers and receivers, transistor modeling, and cryogenic electronics.

Evan C. Knox-Davies received his BEng and MScEng degrees in electronic engineering from the University of Stellenbosch in 1996 and 1999, respectively, and his PhD from the University of Surrey in 2006. From 2010 onward, he worked for EMSS Antennas on the cryogenic receivers for the MeerKAT radio telescope, his main focus being the development of RF testing systems suitable for production quantities. Since 2016, he has mainly been involved with development of radar systems for sport.

Sarel J. Marais is the co-founder of Inrange Golf, which applies radar tracking technology to sports entertainment and training applications. Prior to that, he was involved in the design and development of the receiver front-end systems for the MeerKAT radio telescope at EMSS Antennas. He received his BEng and MEng degrees in electronic engineering from the University of Stellenbosch in 2006.

Isak P. Theron is the director of radio astronomy at EMSS Antennas. He received his BEng and MEng degrees in electronic engineering, both cum laude, and the $\mathrm{PhD}$ (working on wave propagation in anisotropic chiral media) from the University of Stellenbosch in 1989, 1991, and 1995, respectively. Currently, he is primarily involved in the design of antennas and cryogenic feed systems for radio astronomy. 